\title{
Targeting Membrane HDM-2 by PNC-27 Induces Necrosis in Leukemia Cells But Not in Normal Hematopoietic Cells
}

\author{
ANUSHA THADI ${ }^{1}$, LAUREN LEWIS $^{1}$, EVE GOLDSTEIN ${ }^{1}$, ANSHU AGGARWAL ${ }^{1}$, MARIAN KHALILI ${ }^{1}$, \\ LINDSAY STEELE ${ }^{1}$, BORIS POLYAK ${ }^{1}$, SHABNAM SEYDAFKAN ${ }^{2}$, MARTIN H. BLUTH ${ }^{3}$, KRISTINE A. WARD ${ }^{4}$, \\ MICHAEL STYLER ${ }^{5}$, PAUL M. CAMPBELL ${ }^{6}$, MATTHEW R. PINCUS ${ }^{2 *}$ and WILBUR B. BOWNE ${ }^{1,7 *}$ \\ ${ }^{1}$ Division of Surgical Oncology, Department of Surgery, \\ Drexel University College of Medicine, Philadelphia, PA, U.S.A.; \\ ${ }^{2}$ Department of Pathology and Laboratory Medicine, SUNY Downstate Medical Center, Brooklyn, NY, U.S.A.; \\ ${ }^{3}$ Department of Pathology, Wayne State University School of Medicine, Detroit, MI, U.S.A.; \\ ${ }^{4}$ Department of Hematology and Oncology, Leukemia Program, \\ University of Pennsylvania, Philadelphia, PA, U.S.A; \\ ${ }^{5}$ Department of Hematology and Oncology, Bone Marrow Transplant Program, \\ Fox Chase Cancer Center, Philadelphia, PA, U.S.A.; \\ ${ }^{6}$ The Marvin and Concetta Greenberg Pancreatic Cancer Institute, \\ Cancer Biology Program, Fox Chase Cancer Center, Philadelphia, PA, U.S.A.; \\ ${ }^{7}$ Department of Surgery, Thomas Jefferson University, Sidney Kimmel Medical College, Philadelphia, PA, U.S.A.
}

\begin{abstract}
Background/Aim: Anticancer peptide PNC-27 binds to HDM-2 protein on cancer cell membranes inducing the formation of cytotoxic transmembrane pores. Herein, we investigated HDM-2 membrane expression and the effect of PNC-27 treatment on human non-stem cell acute myelogenous leukemia cell lines: U937, acute monocytic leukemia; OCI-AML3, acute myelomonocytic leukemia and HL60, acute promyelocytic leukemia. Materials and Methods: We measured cell surface membrane expression of HDM-2 using flow cytometry. Cell viability was assessed using MTT assay while direct cytotoxicity was measured by lactate dehydrogenase $(\mathrm{LDH})$ release and induction of apoptotic markers annexin V and caspase-3. Results: HDM2 is expressed at high levels in membranes of U937, OCIAML3 and HL-60 cells. PNC-27 can bind to membrane HDM-2 to induce cell necrosis and LDH release within $4 \mathrm{~h}$. Conclusion: Targeting membrane HDM-2 can be a potential strategy to treat leukemia. PNC-27 targeting membrane
\end{abstract}

This article is freely accessible online.

Correspondence to: Wilbur B. Bowne, MD, Thomas Jefferson University Hospital, 1100 Walnut Street, Philadelphia, PA 19107, U.S.A. Tel: +1 2155036855, Fax: +1 215503850, e-mail: Wilbur.Bowne@jefferson.edu; Matthew R. Pincus, MD, Ph.D., SUNY Downstate Medical Center, Brooklyn, NY 11203, U.S.A. Tel: +1 7184394162, Fax: +1 7184394162, e-mail: mrpincus2010@gmail.com

Key Words: Cancer cell membrane, leukemia, PNC-27, HDM-2.
HDM-2 demonstrated significant anti-leukemia activity in a variety of leukemic cell lines.

Advances in cancer research have shown that human double minute 2 (HDM-2), an oncoprotein known to regulate tumor suppressor p53, is uniquely expressed in the membranes of solid and non-solid tumor cells independent of their p53 status $(1,2)$. The membrane localization and expression of HDM-2 is highly specific to transformed cells and hence, it is an attractive therapeutic target for cancer treatment (2-4). Acute myeloid leukemia (AML) is a hematological malignancy characterized by heterogeneous sub-populations of primitive and more differentiated clonal cells: a spectrum of cancer cells, which contributes to treatment-refractory conditions and poor prognosis (5). Off target cytotoxicity of currently available chemotherapies for acute leukemias is a major obstacle and there is a need for novel treatment strategies demonstrating minimal to no cytotoxicity towards nonmalignant cells $(6,7)$.

PNC-27 is a peptide construct developed to prevent the interaction of human HDM-2/mouse MDM-2 and p53. PNC27 is composed of amino acid residues 12-26 of the HDM-2 binding domain of $\mathrm{p} 53$ on its amino terminus and a membrane residency peptide (MRP) on its carboxyl terminus $(8,9)$. This peptide co-localizes with HDM-2 that is uniquely expressed in the membranes of a wide variety of solid tissue cancer cells but not in normal (8-10) cells and induces the formation of transmembrane pores causing p53-independent tumor, but not normal, cell necrosis $(3,4,11)$. 
More recently, PNC-27 has further been found to induce necrosis of leukemia cell lines including a chronic myeloid leukemia (CML) cell line (3) and acute myelogenous (myeloblastic) leukemia (AML) cells from patients that are enriched with stem cells (2). In the latter study (2), PNC-27 was found to be cytotoxic to leukemia stem cells (LSCs) and to co-localize with membrane-bound HDM-2 in leukemia cell membranes such that the extent of membrane HDM-2 expression correlated with the $\mathrm{IC}_{50}$ for PNC-27-induced cell killing (2). Furthermore, PNC-27 was found to induce AML stem cell killing in vivo (2). Moreover, this study demonstrated a significantly higher membrane HDM-2 expression in $\mathrm{CD} 34^{+}$AML blast subpopulations as compared to CD34- AML blast subpopulations (2).

Our aim was to extend these studies to additional subtypes of myelogenous leukemia cells with different phenotypical and genetic characteristics including more differentiated subpopulations exhibiting CD34 negativity and different p53 status. In this study, we selected three non-stem cell (CD34-) AML cell lines corresponding to three different FAB classification types: M3 (HL60 cells), M4 (OCI-AML3) and M5 (U937). Herein, we detected high levels of HDM-2 expression in the membranes of these cells and observed a different treatment efficacy than that previously reported for AML stem cells when targeting HDM-2 with PNC-27 (2). Importantly, we show no PNC-27 effect on normal hematopoietic cells. These studies further elucidate the mechanism of cell death and suggest that PNC-27 may be an effective agent in treating leukemia.

\section{Materials and Methods}

Peptides. Peptides were synthesized using solid phase method with $>95 \%$ HPLC purity. PNC-27 and the negative control peptide, PNC29 were obtained from Biopeptides Corp (San Diego, CA, USA). PNC-27 is derived from the HDM-2 binding domain of p53 and PNC-29 is derived from cytochrome p450 with a membrane residency peptide, MRP sequence (shown in italics) attached to their carboxyl terminal (8-10).

\section{PNC-27: PPLSQETFSDLWKLLKKWKMRRNQFWVKVQRG PNC-29: FSTGKRIMLGE $K K W K M R R N Q F W V K V Q R G$}

Cell lines. All cell lines used in this study were CD34-. Human U937, a monocytic leukemia cell line (FAB classification, M5) (12) was a gift from Dr. Shendra Passic, Drexel University College of Medicine and Dr. Pasun K. Datta, Temple University, Philadelphia, PA, USA. These cells were maintained in RPMI-1640 with Lglutamine (VWR International, Radnor, PA, USA) plus 10\% fetal bovine serum (FBS) and 1\% penicillin/streptomycin. Human OCIAML3 cells, a myelomonocytic leukemia cell line [FAB classification, M4, (13)], were a gift from Dr. Ellen Lori Weisberg, Dana-Farber Cancer Institute, Boston, MA, USA and were grown in Alpha MEM Eagle (Lonza, Alpharetta, GA, USA) plus 20\% FBS and $1 \%$ penicillin/streptomycin. Human HL60 cells, a promyelocytic leukemia cell line (FAB classification, M3) and
Iscove's Modified Dulbecco's Medium (IMDM) were obtained from American Type Culture Collection (ATCC, Manassas, VA, USA) and these cells were maintained in IMDM plus 10\% FBS and 1\% penicillin/streptomycin. Cells were cultured in a humidified incubator at $37^{\circ} \mathrm{C}$ and $5 \% \mathrm{CO}_{2}$.

Rat mononuclear cells (rat MNCs). Blood was collected at exsanguination from Lewis rats through inferior vena cava cannulation (IVC) into sodium heparin coated tubes using a standard procedure by a protocol approved by Drexel University Animal Care and Use Committee. Blood was mixed 1:1 with RPMI-1640 containing 15 units of heparin per ml. Histopaque-1083 (Sigma Aldrich, St. Louis, MO, USA) density gradient centrifugation was used to collect buffy coat, and isolate rat mononuclear cells. Mononuclear cells were then suspended in RPMI-1640 supplemented with $10 \% \mathrm{FBS}$ and $1 \%$ penicillin/streptomycin.

Flow cytometry. Cells were grown to confluence, harvested, and washed with chilled Dulbecco's phosphate buffered saline (DPBS) without $\mathrm{Ca}^{2+} \& \mathrm{Mg}^{2+}$. Cells were then suspended in chilled DPBS containing $10 \%$ FBS and $2 \mathrm{mM}$ EDTA at a concentration of $1 \times 10^{6}$ cells/ ml. Cells were blocked and incubated with polyclonal rabbit anti-HDM-2 (1:20) or IgG isotype control (1:20) for $1 \mathrm{~h}$. After 3 washes, cells were incubated with goat anti-rabbit IgG conjugated to DyLight ${ }^{\circledR} 650$ (1:250) for $1 \mathrm{~h}$. Cells were then washed to remove unbound antibody and stained with $5 \mu \mathrm{l}$ of $10 \mu \mathrm{g} / \mathrm{ml}$ propidium iodide (PI). HDM-2 expression on the cell surface was analyzed using BD AccuriTM C6 cytometer system and compared to unstained or isotype stained cells. We identified the percentage of cells positively stained with HDM-2 and calculated median fluorescence from each group of cells using flow cytometry.

Cell viability assay. Cancer cells were treated with increasing concentrations of PNC-27 and PNC-29 in 24 well plates. Rat mononuclear cells were treated with increasing concentrations of PNC-27 and with $100 \mu \mathrm{M}$ of PNC-29 in 24 well plates. After $4 \mathrm{~h}$, cell viability was tested using 3-[4,5-dimethylthiazol-2yl]-2,5diphenyl tetrazolium bromide (MTT) assay kit (Promega, Madison, WI, USA).

Confocal microscopy. Cells were seeded at a density of $2 \times 10^{6}$ cells per $2 \mathrm{ml}$ media per well in a 6 well plate and grown overnight. Cells were then incubated with PNC-27 or PNC-29 at a concentration of $4 \mu \mathrm{M}$ for U-937 cells, $60 \mu \mathrm{M}$ for OCI-AML3 cells and $80 \mu \mathrm{M}$ for HL-60 cells for $1 \mathrm{~h}$. These peptide concentrations were determined from the viability assays. Cells were washed with $1 \mathrm{X}$ DPBS and fixed with $4 \%$ formaldehyde in $1 \mathrm{X}$ DPBS for $10 \mathrm{~min}$ at $37^{\circ} \mathrm{C}$. Blocking was performed at room temperature for $1 \mathrm{~h}$ with $1 \mathrm{X}$ DPBS containing $2 \%$ bovine serum albumin (BSA). Cells were treated with a mixture of polyclonal rabbit anti-MDM-2 (AF1244, Novus Biologicals, Centennial, CO, USA) [1:10] and monoclonal mouse anti-p53 (DO-1, Santa Cruz Biotechnology, Santa Cruz, CA, USA) [1:40] overnight on a shaker at $4^{\circ} \mathrm{C}$. After 3 washes with $1 \mathrm{X}$ DPBS, cells were incubated with secondary antibodies, the polyclonal antirabbit red fluorophore (DyLight ${ }^{\circledR}$ 650) (Abcam Inc., Cambridge, MA, USA) [1:200] and polyclonal anti-mouse green fluorophore (DyLight ${ }^{\circledR} 488$ ) (Abcam Inc.) [1:200] for $1 \mathrm{~h}$ at room temperature protected from light. Cells were washed with $1 \mathrm{X}$ DPBS containing $0.1 \%$ tween 20 and suspended in $1 \mathrm{X}$ DPBS $\left(0.5 \times 10^{6}\right.$ cells $\left./ 1 \mathrm{ml}\right)$. Cells were transferred to glass slides using cytospin at $800 \mathrm{rpm}$ for 
A. $U 937$

I.

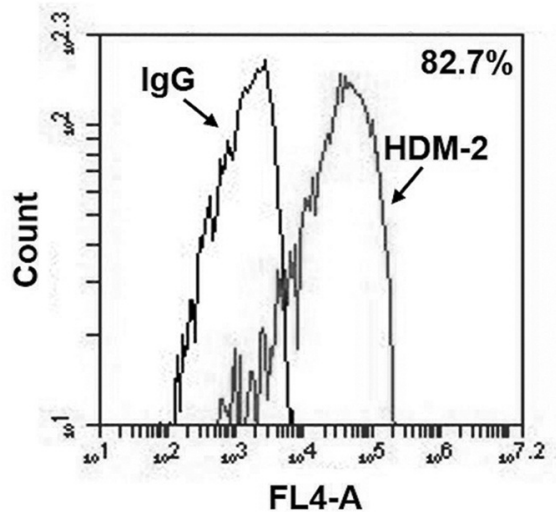

II.

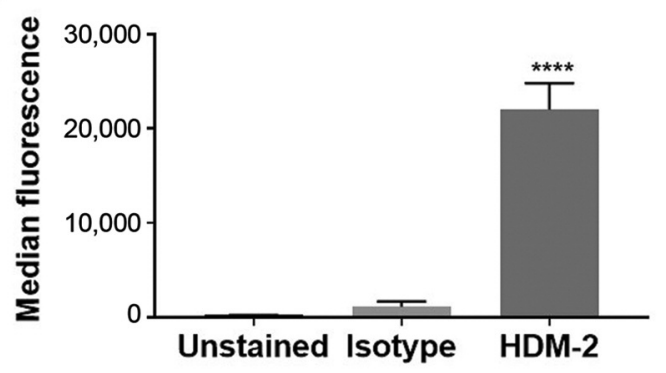

C. HL60

I.

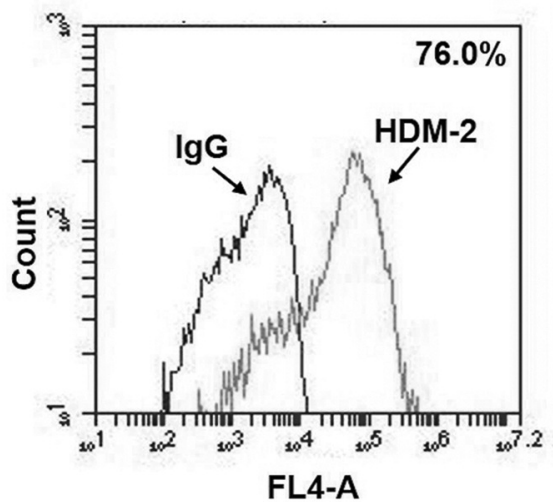

II.

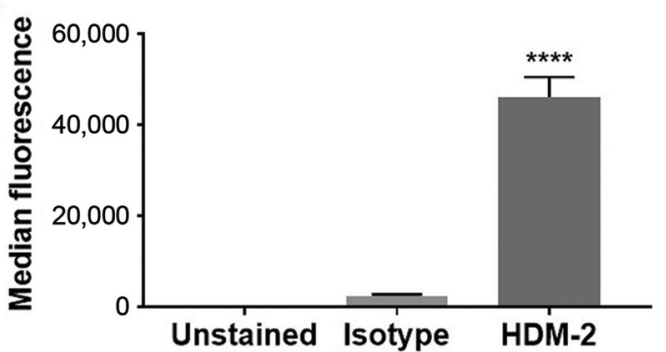

B. OCI-AML3

I.

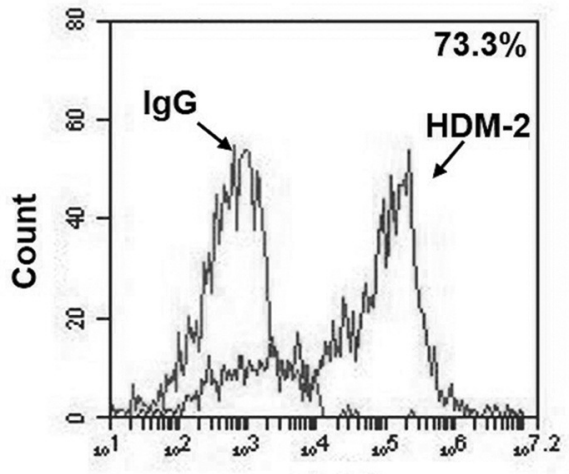

FL4-A

II.

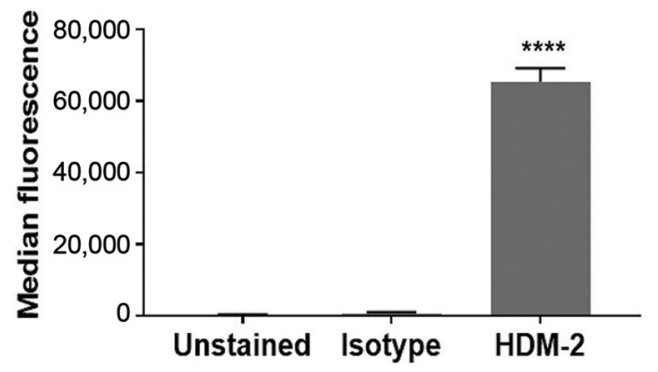

D. RAT MONONUCLEAR CELLS

I.

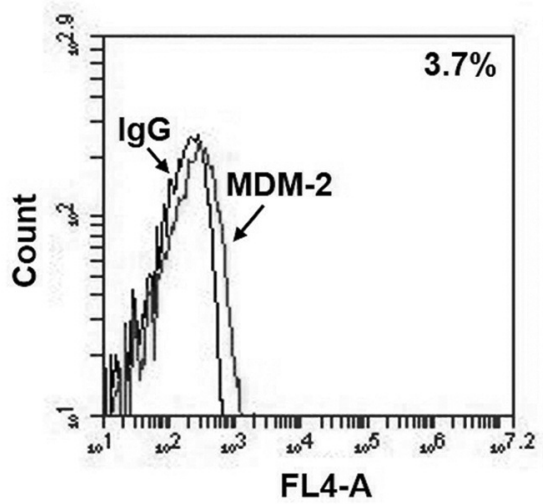

II.

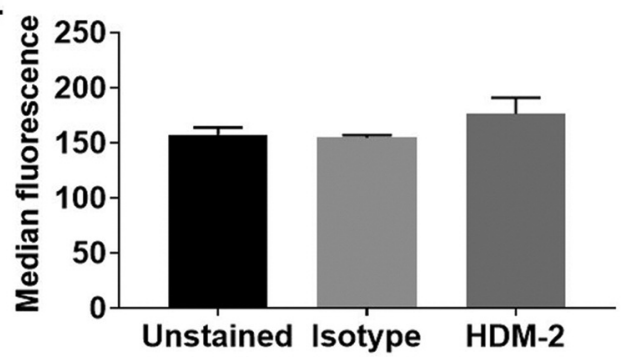

Figure 1. U937, OCI-AML3 and HL60 cells express high levels of HDM-2 on their cell membranes. Flow cytometry data was obtained from 3 independent experiments. Cells were stained with rabbit anti-HDM-2 labeled with anti-rabbit-DyLight ${ }^{\circledR} 650$ or isotype (IgG) to identify cells that possess membrane $H D M-2$. In order to eliminate background, cells were unstained or stained with secondary antibody only. In addition, cells were stained with propidium iodide to select for live cells and eliminate dead cells. Live A. U937, B. OCI-AML3, C. HL60 and D. rat mononuclear cells (rat MNCs) expressing MDM2 on their intact membranes as a percentage of all stained cells (A.I, B.I, C.I and D.I) and expression of H/MDM-2 as median fluorescence from different groups of cells (A.II, B.II, C.II and D. II). Data are shown as median fluorescence \pm SEM. ****p $\leq 0.0001$ in comparison to isotype control cells. 

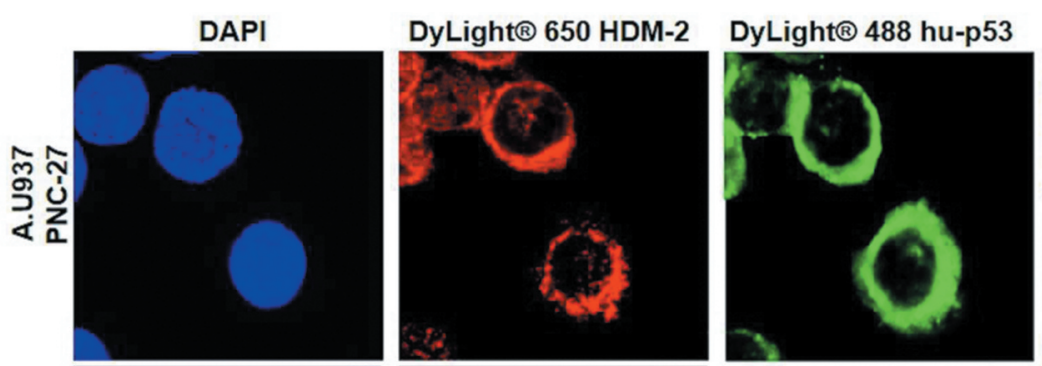

\section{Colocalization}
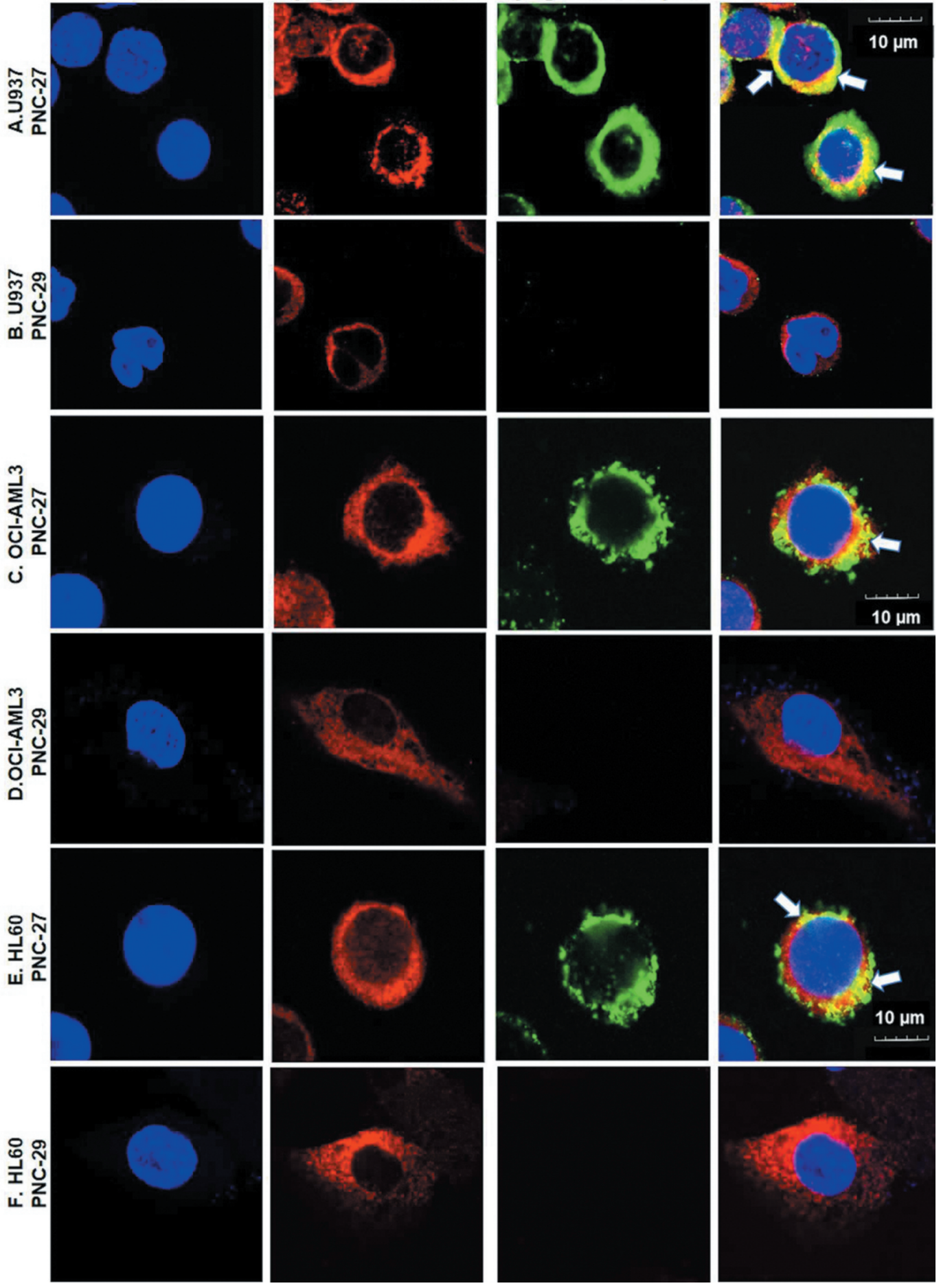

Figure 2. HDM-2 is expressed in leukemia cell membrane and PNC-27 colocalizes with the membrane HDM-2. Confocal fluorescence microscopy of A. U937, C. OCI-AML3 and E. HL60 cells indicated the presence of membrane HDM-2 and displayed its association with PNC-27 when leukemia cells were treated with PNC-27 for 1h. To visualize membrane HDM-2 in red (second column), cells were stained with rabbit anti-HDM-2 followed by incubation with red fluorescent DyLight ${ }^{\circledR} 650$ conjugated anti-rabbit. Third column shows PNC-27 staining in green when PNC-27 treated cells were incubated with mouse anti-human (hu)-p53 followed by green fluorescent DyLight ${ }^{\mathbb{B}} 488$ conjugated anti-mouse. Yellow fluorescence produced by the overlap of red and green signals at the membrane indicated by white arrows (fourth column) demonstrates colocalization of PNC-27 and HDM-2 on the cell membrane. B, D and F represent U937, OCI-AML3 and HL60 treated with PNC-29 for $1 \mathrm{~h}$. There is no colocalization in PNC-29 treated cells as the cells are positively stained with red rabbit anti-HDM-2 only. Cell nuclei were counterstained with blue DAPI and images were captured at 100x magnification. Scale bar, $10 \mu \mathrm{m}$. 
A.U937 MTT, 4h

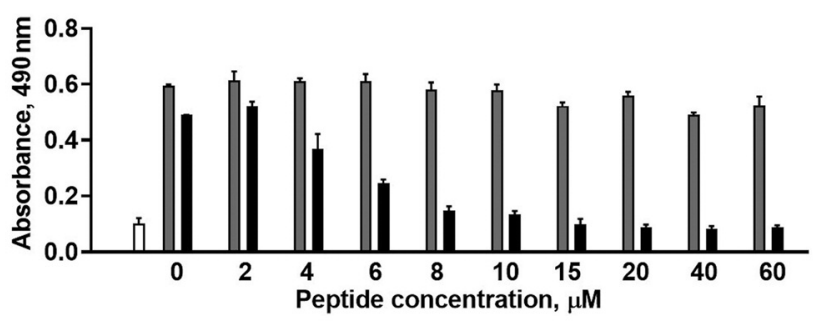

C.HL60 MTT, 4h

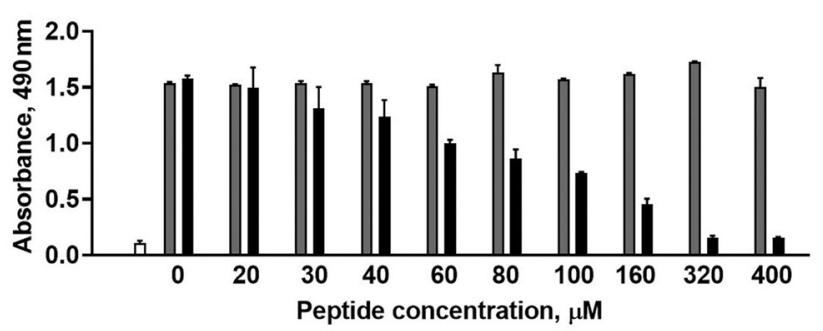

B.OCI-AML3 MTT, 4h

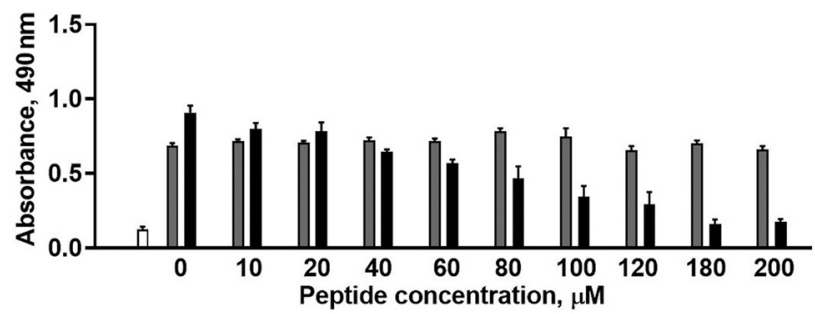

D.RAT MONONUCLEAR CELLS MTT, $4 \mathrm{~h}$

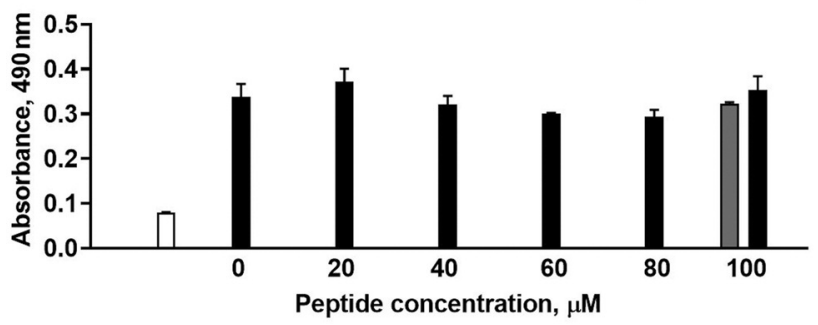

$\square$ Lysis buffer

PNC-27

PNC-29

Figure 3. Anti-leukemic activity of PNC-27 increases in a dose dependent manner. Leukemia cells were incubated with PNC-27 and PNC-29 (negative control) and cell viability was examined using MTT assay after $4 \mathrm{~h}$. Cells were lysed with lysis buffer (positive control) to induce maximum cell lysis. PNC-27 reduced viability of A. U937, B.OCI-AML3 and C. HL60 in a dose dependent manner. PNC-29 did not induce any cytotoxicity in leukemia cells. PNC-27 and PNC-29 showed no cytotoxicity towards D. normal rat mononuclear cells. Results derived from three independent experiments and shown as mean absorbance \pm SEM.

A.U937 LDH, 4h

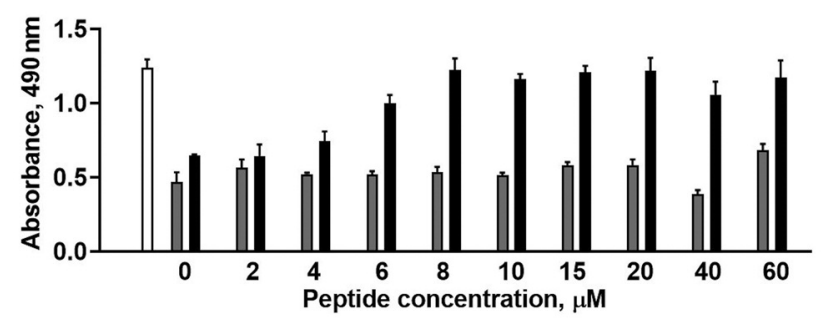

C.HL60 LDH, $4 \mathrm{~h}$

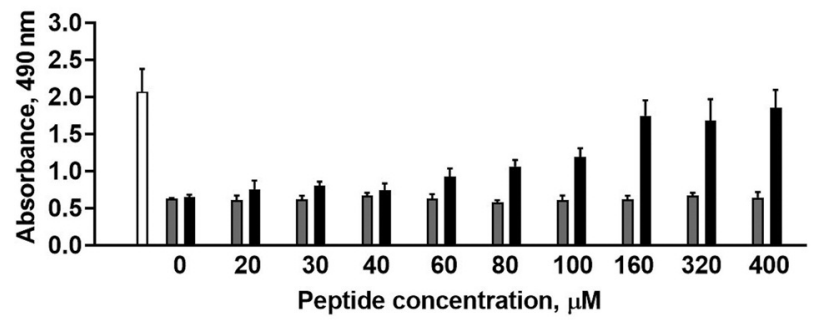

B.OCI-AML3 LDH, 4h

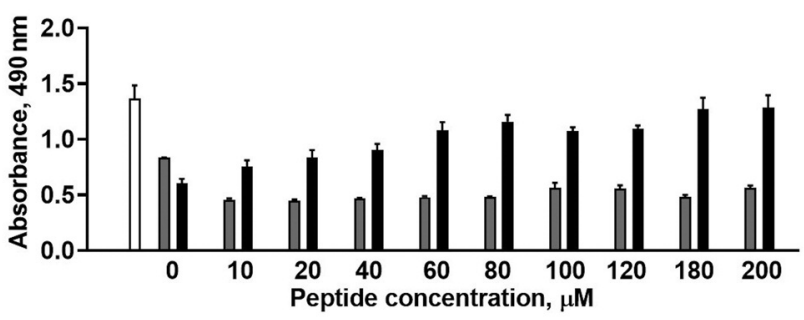

D.RAT MONONUCLEAR CELLS LDH, 4h

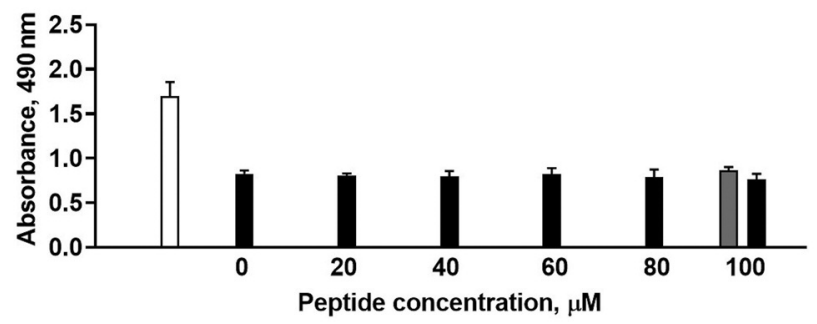

$\square$ Lysis buffer

PNC-27

Figure 4. Anti-leukemic activity of PNC-27 occurs by tumor cell necrosis. A. U937, B. OCI-AML3, C. HL60, and D. normal rat mononuclear cells were incubated with different concentrations of PNC-27 and PNC-29. Incubation of cells with lysis buffer resulted in maximum LDH release in culture media. $P N C-27$ treatment resulted in LDH release in a dose dependent manner. No LDH release was observed in leukemia cells incubated with PNC-29 and D. rat mononuclear cells incubated with PNC-27 or PNC-29. Experiments were performed in triplicate, and data are shown as mean absorbance \pm SEM. 

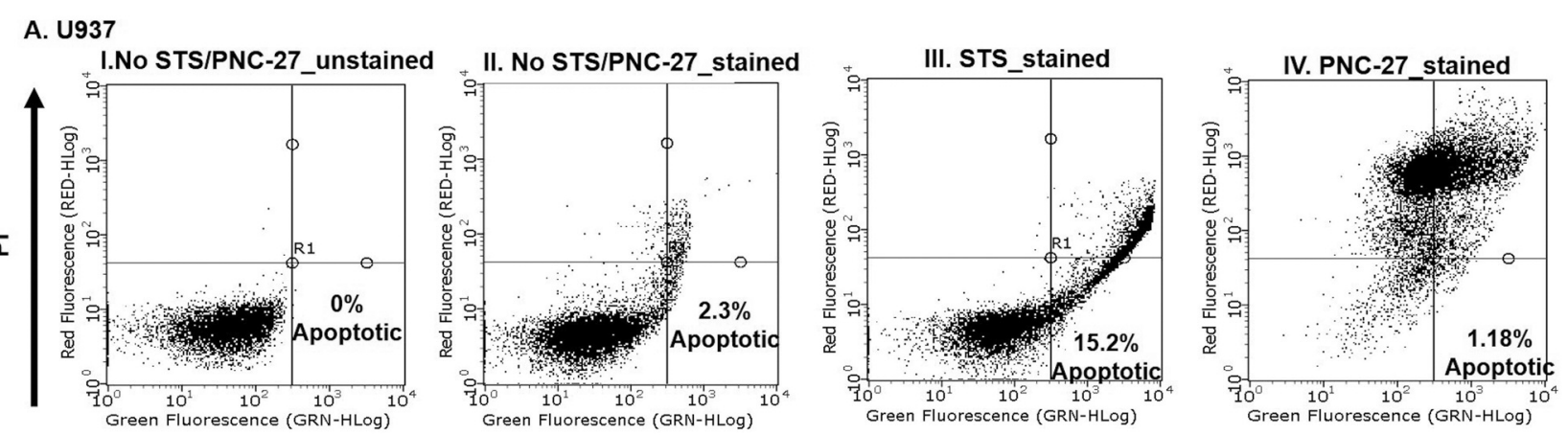

Annexin V or Apoptotic

V.

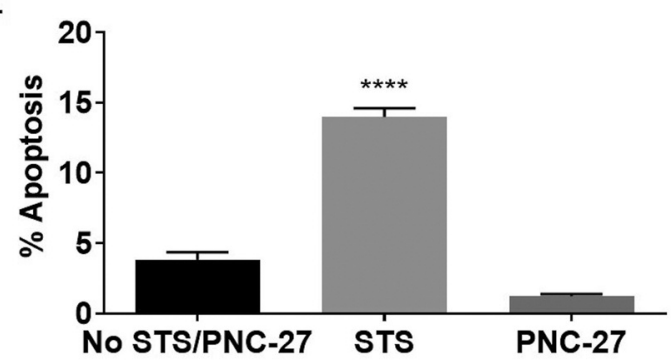

B. OCI-AML3
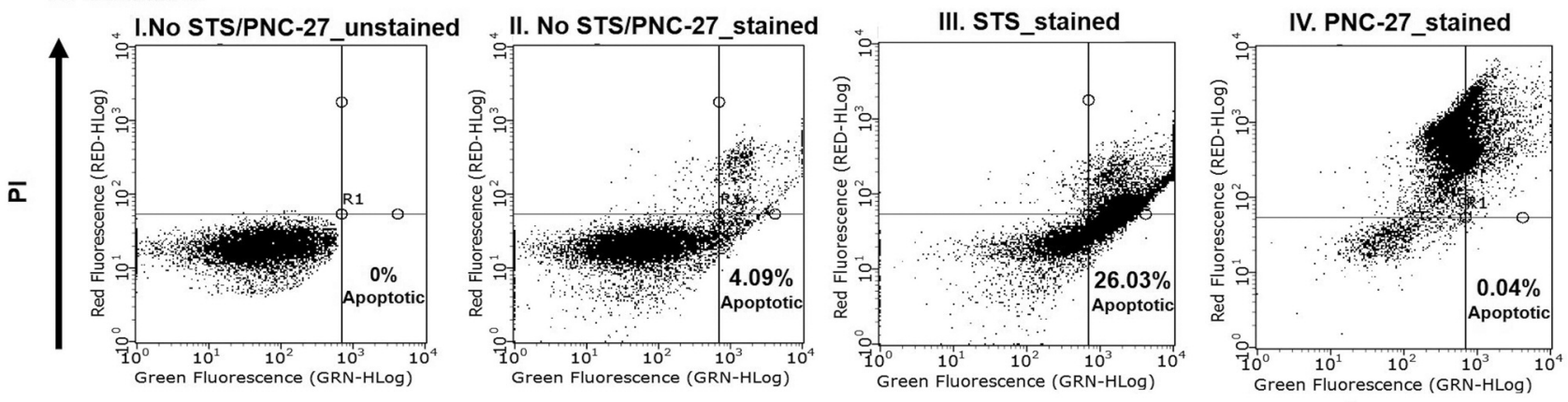

Annexin V or Apoptotic

V.

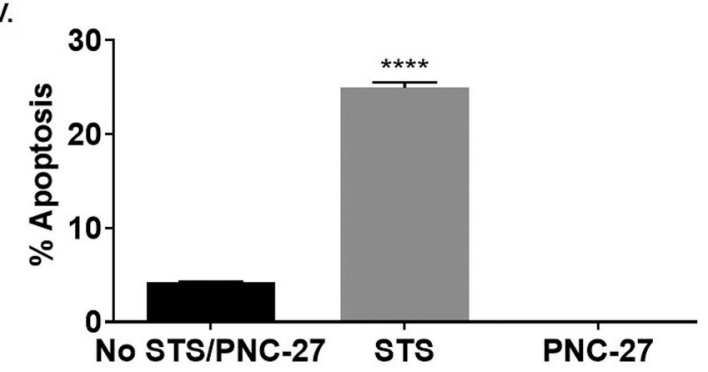

Figure 5. Continued

6 min. Cells were then stained with DAPI, covered with a cover slip and visualized using an OLYMPUS FLUOVIEW FV3000 microscope (Olympus, Waltham, MA, USA) at $100 \times$ magnification. Scale bars were added using cellSens software.
Necrotic and apoptotic assays. All cell lines were incubated with PNC-27 or PNC-29. The levels of the necrotic marker lactate dehydrogenase $(\mathrm{LDH})$ in media were determined $4 \mathrm{~h}$ post-treatment using CytoTox96 assay (Promega). 


\section{HL60}
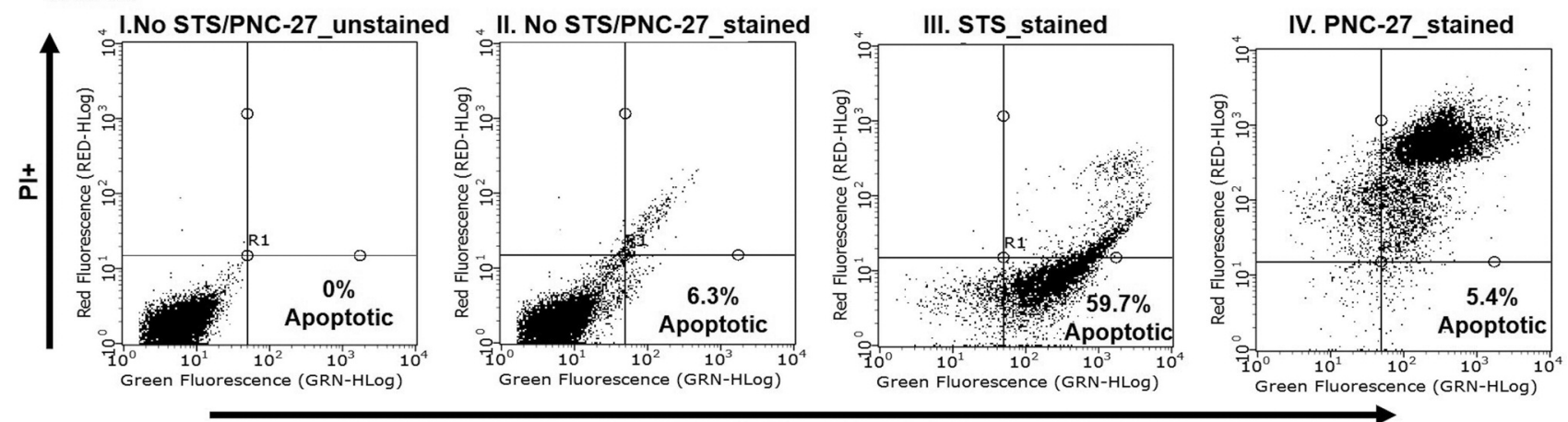

Annexin V+ or Apoptotic

V.

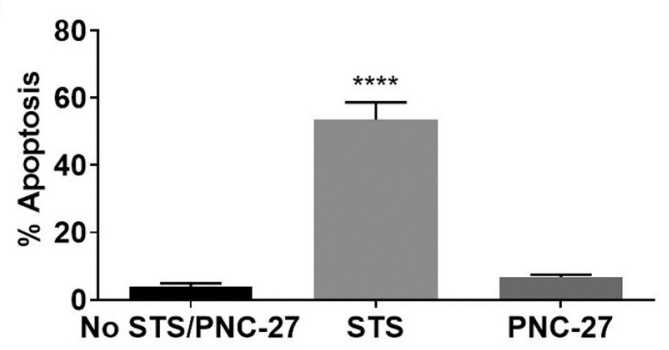

Figure 5. Anti-leukemic activity of PNC-27 did not result in early apoptosis as measured by flow cytometry experiments. Early apoptosis was estimated by annexin V/PI staining of cells (see Methods section). A. U937 were treated with $5 \mu M$ PNC-27 or $4 \mu M$ staurosporine (STS), B. OCI-AML3 were treated with $85 \mu M$ PNC-27 or $4 \mu M$ STS whereas C. HL60 were treated with $90 \mu M$ PNC-27 or $10 \mu M$ STS for 1h. Frames I in Figures 5 A, B and $C$ show the cytometric pattern of untreated and unstained cells. Frames II in Figures 5 A, B and C show the cytometric pattern of untreated cells stained with the annexin V/PI stain showing basal levels of annexin V positive or early apoptotic cells. Frames III in Figures 5 A, B and C show the cytometric pattern of cells treated with STS only and then stained with the annexin V/PI stain and show that STS induced significant apoptosis as indicated by high levels of cells positively stained for annexin V. Frames IV in Figures 5 A, B and C show the cytometric pattern of cells treated with PNC-27 only and then stained with the annexin V/PI stain and show that PNC-27 treatment resulted in basal levels of annexin V positive cells comparable to no treatment. Frames V in Figures 5 A, B and C are bar graphs that demonstrate the percent of apoptosis (number of early apoptotic cells as a percentage of all stained cells in the group) induced in the three groups: I. No treatment with STS/PNC-27, II. STS treated and III. PNC27 treated. Results are shown as \% apoptosis \pm SEM from four independent experiments. $* * * * \leq 0.0001$ versus untreated stained cells.

Cells were grown to $60-70 \%$ confluence and treated with PNC27 or staurosporine (STS), an established apoptosis inducer. U-937, OCI-AML3 and HL60 cells were treated with $5 \mu \mathrm{M}, 85 \mu \mathrm{M}$ and 90 $\mu \mathrm{M}$ of PNC-27, respectively. As a positive control of apoptotic induction, U937 and OCI-AML3 were treated with $4 \mu \mathrm{M}$ STS whereas HL-60 were treated with $10 \mu \mathrm{M}$ STS. The early apoptotic marker annexin $\mathrm{V}$ was measured at $4 \mathrm{~h}$ and the late apoptotic marker caspase-3/CPP32 was measured at $6 \mathrm{~h}$ after treatment with PNC-27. To determine annexin $\mathrm{V}$ positive cells, the annexin $\mathrm{V}$ FITC/ propidium iodide (PI) assay kit from BD Biosciences (San Jose, CA, USA) and Guava InCyte flow cytometer system were used to stain cells and acquire the data. Caspase-3/CPP32 activity after $6 \mathrm{~h}$ was determined using the Caspase-3/CPP32 colorimetric assay kit (BioVision Inc., Milpitas, CA, USA).

Computational and statistical methods. GraphPad Prism 8.0 (GraphPad Software, La Jolla, CA, USA) was used to analyze all quantitative assay results from flow cytometry, confocal microscopy, MTT assays and LDH assays. Results are shown as means \pm 1 standard error of the mean (SEM). Statistical significance between assay values was determined using the two-tailed Student's $t$-test. $p$-Values $<0.05$ were considered to indicate statistically significant difference. $\mathrm{IC}_{50}$ values for each peptide were determined using nonlinear regression curve fit.

\section{Results}

Membrane HDM-2 is selectively expressed at high levels in human leukemia U937, OCI-AML3 and HL60 cells. We examined the expression of HDM-2 on the cell surface of CD34- U937, OCI-AML3 and HL60 using flow cytometry. Human monocytes do not grow well in vitro (unpublished observation and personal communication). Hence, we chose normal rat mononuclear cells as negative control cells and compared the expression of HDM-2 in these cells to leukemia cells. As shown in Figure 1, a large population of leukemia cells expressed membrane HDM-2 and we noticed a considerable shift in median fluorescence of HDM-2 in 
leukemia cells compared to their isotype control ( $\mathrm{IgG})$. In contrast, there was a negligible shift in median fluorescence of HDM-2 in rat mononuclear cells compared to isotype control groups. This indicates significant expression of membrane HDM-2 in the tested human leukemia cells but not in normal rat mononuclear cells (MNCs).

PNC-27 binds to membrane HDM-2 in vitro. To test if membrane HDM-2 can colocalize with PNC-27, cells were treated with PNC-27 for $1 \mathrm{~h}$. Cells were then incubated with rabbit anti-HDM-2 fluorescently labeled with red anti-rabbit antibody and mouse anti-p53 fluorescently labeled with green anti-mouse antibody. If the two signals are localized in the same area, yellow fluorescence is emitted indicating colocalization.

Confocal images of U937, OCI-AML3 and HL60 (Figure $2 \mathrm{~A}, \mathrm{~B}$ and $\mathrm{C}$ ) further confirmed the expression of HDM-2 in the membrane by the significant red signal emitted due to localization of red anti-HDM2 in the membrane. Similarly, significant green fluorescence was observed at the cell surface as a result of PNC-27 localization in the membrane. It should be noted here that U937 and HL60 are p53 null cell lines and hence, the green fluorescence emitted is completely from the interaction between green anti-p53 and PNC-27 (DO-1 anti-p53 binds to PNC-27, a peptide construct derived from p53) on the cell surface. Furthermore, even though OCI-AML3 has wildtype p53, p53 is not stable at the protein level and expresses no significant levels of p53 protein (14). Hence, we can conclude that the green fluorescence in OCIAML3 cells is a result of PNC-27 localized at the cell membrane. We observed strong binding of HDM-2 and PNC-27 as evidenced by significant yellow fluorescence at the cell surface. This confirms that PNC-27 can bind to membrane HDM-2 of human leukemia cells. Absence of green fluorescence in PNC-29 treated U937, OCI-AML3 and HL60 cells indicated that the green anti-p53 antibody staining is specific to PNC-27.

PNC-27 is selectively cytotoxic to human leukemia cells but not to normal rat mononuclear cells. MTT assay was used to assess the impact of PNC-27/HDM-2 association on cell viability. We noticed a dose dependent decrease in cell viability of U937, OCI-AML3 and HL60 cells after $4 \mathrm{~h}$. PNC-27 induced cell death in U937 with greater efficiency at very low $\mathrm{IC}_{50}$ of $4.7 \mu \mathrm{M}$ as compared to the other two AML cell lines. The $\mathrm{IC}_{50}$ s for OCI-AML3 and HL60 were 83.9 $\mu \mathrm{M}$ and $91.1 \mu \mathrm{M}$, respectively. In addition, PNC-27 was tested in normal rat MNCs and we observed no PNC-27induced cytotoxicity in normal cells. The negative control peptide PNC-29 did not demonstrate any effect on the viability of cancer and normal cells (Figure 3). Therefore, PNC-27 induces its cytotoxic effects selectively in leukemia cells without any toxicity towards normal mononuclear cells even at a dose higher than the highest $\mathrm{IC}_{50}$ observed. This observation is consistent with our previous studies where we observed PNC-27 induced anti-cancer activity in human leukemia K562 cells while sparing the normal murine lymphoid cells (3).

Necrosis is the mechanism of anti-leukemic activity of PNC27. To investigate the mechanism of cell death, levels of necrotic and apoptotic markers were measured after treatment with different concentrations of PNC-27. After 4 $\mathrm{h}$, there was a dose dependent release of $\mathrm{LDH}$ in culture supernatants which indicates necrotic cell death as shown in Figure 4. In addition, the LDH assay confirmed the absence of PNC-27-mediated cytotoxicity in normal rat mononuclear cells. PNC-29 did not increase LDH levels in the media of cancer or normal cells.

We then studied the effect of PNC-27 on early and late apoptotic markers. Apoptosis begins with exposure of phosphatidylserine on the outer plasma membrane and can be detected by staining the cells with annexin V. Staurosporine (STS), a known inducer of apoptosis, was used as a positive control. There was no significant staining of cells with annexin V, when treated with PNC-27 (Figure 5). Since this early event is reversible, we confirmed the findings by testing the effect of PNC-27 on caspase- 3 activation which is a later apoptotic event. As shown in Figure 6, PNC-27 had no effect on caspase-3 whereas STS induced significant caspase- 3 activity. These observations are consistent with our previous findings in solid and non-solid tumors which suggest that PNC-27 induced cell death by necrosis.

\section{Discussion}

We have previously found that PNC-27 induces tumor cell necrosis, but not apoptosis, in a wide variety of solid tissue tumors $(4,8,10,11)$ while having no effect on the viability or growth of untransformed cells. The tumoricidal effect is due to its ability to rapidly induce trans-membrane pore formation, resulting in extrusion of intracellular contents and cell death. We found that PNC-27 co-localizes in cancer cell membranes with HDM-2 to which it binds in a membrane active amphipathic helix-loop-helix conformation resulting in induction of pore formation (4). In this report, we explored whether PNC-27 produces these same effects on non-solid tissue tumors such as leukemia cells. We therefore extended our studies to include hematopoietic tumors. In particular, we found that PNC-27 killed human chronic myelogenous leukemia (K562) cells without affecting normal hematopoietic cells (3) but we did not investigate its mechanism of action on these cells.

In this study, we expanded our investigations to determine whether PNC-27 kills other types of leukemia cells in the 

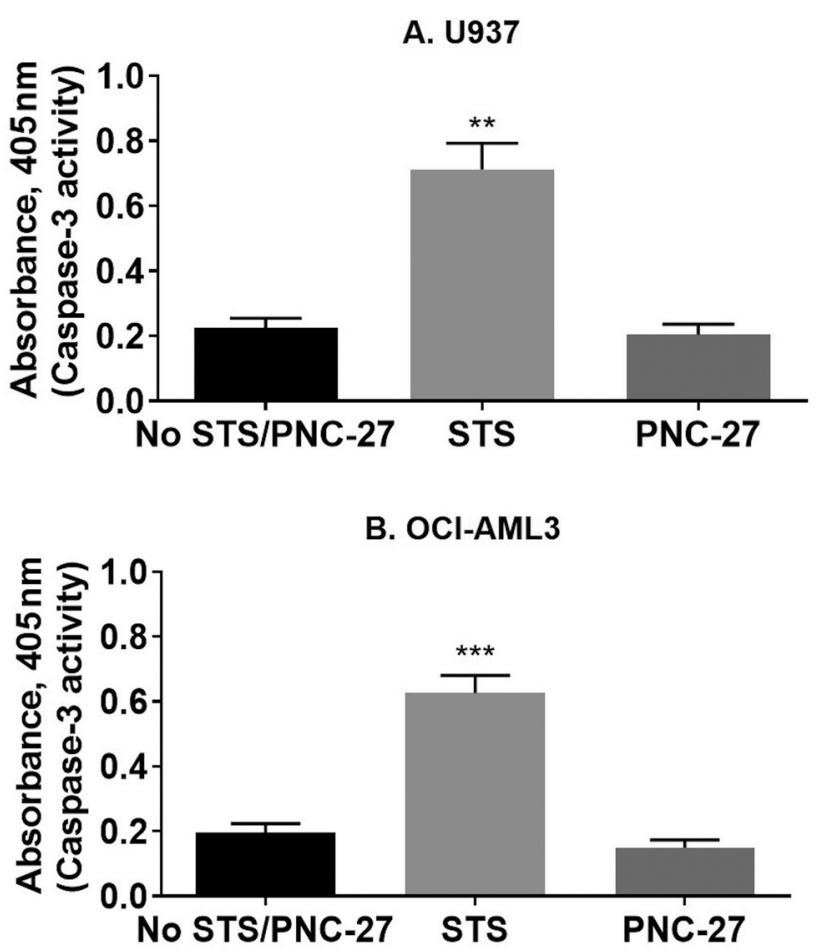

C. HL60

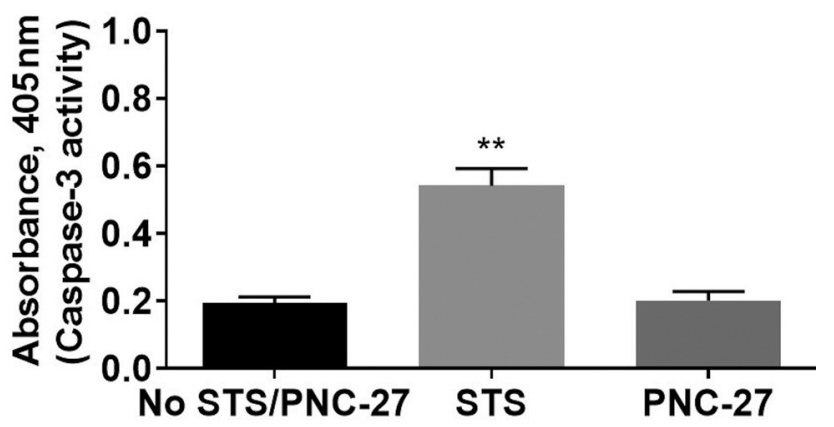

Figure 6. PNC-27 mediates its anti-leukemic activity without induction of late apoptotic events. Increase in caspase-3 activity is an indication of late apoptotic cells. Caspase-3 activity was estimated after A. U937 cells were treated with $5 \mu M$ PNC-27 or $4 \mu M$ STS, B. OCI-AML3 cells were treated with $85 \mu M P N C-27$ or $4 \mu M$ STS and C. HL60 cells were treated with $90 \mu M$ PNC-27 or $10 \mu M$ STS for 6h. STS induced significant levels of caspase-3 activity compared to untreated cells whereas PNC-27 did not induce any caspase-3 activity. Data shows mean absorbance $\pm S E M$ from three independent experiments. ${ }^{*} p \leq 0.01$ and $* * * p \leq 0.001$ versus untreated cells.

myeloid series and, if so, to determine if it does so by a similar mechanism. We first noted that a recent study on this question had been performed using AML cells from ten different patients that contained high fractions of tumor stem cells $\left(\mathrm{CD}^{+} 4^{+}\right)(2)$. These studies indicated that PNC-27 induces tumor cell necrosis and not apoptosis in a dosedependent manner. They further found that there was a good correlation between the extent of expression of HDM-2 in the AML cell membranes and the $\mathrm{IC}_{50}$ of PNC-27. Importantly, this study included the in vivo effects of PNC27 after AML cells from each patient were grafted into the bone marrow of nude mice. Treatment of the mice for three weeks with PNC-27 reduced the elevated white blood cell counts to normal levels and significantly prolonged survival of these mice with no off-target effects compared with the control group treated with the inactive peptide, PNC-29. Transplantation of the bone marrow cells of the treated mice into untreated mice resulted in no increase of white cell counts, further supporting the efficacy of PNC-27 treatment.

In our current study, we used a variety of different CD34negative AML cells to explore whether PNC-27 can induce tumoricidal effects generally with leukemias. For this purpose, we selected three cells lines that differ in genetic background and aggressiveness to be used in this study.

In agreement with a recent study (2), we found that PNC27 co-localizes with HDM-2 in leukemia cell membranes and induces rapid leukemia cell necrosis in a dose-dependent manner. In the previous study, PNC-27 was found not to affect normal bone marrow stem cells, which was in agreement with our earlier findings that a closely related shorter anti-cancer peptide, PNC-28, had no effect on the abilities of hematopoietic stem cells isolated from human cord blood to differentiate into stable derivative cell lines in the presence of growth factors (8). In the present study we also confirmed that PNC-27 had no effect on normal rat mononuclear cells, demonstrating that PNC-27 kills cancer cells with no damage to normal cells.

Furthermore, the range for our $\mathrm{IC}_{50}$ values for leukemia cell killing, i.e., $4.7 \mu \mathrm{M}$ for U937 (myelomonocytic leukemia with histiocytic features) cells to $91.1 \mu \mathrm{M}$ for HL60 (promyelocytic leukemia) cells was similar to the range in the previous study, i.e., 11- $60 \mu \mathrm{M}$ (2). However, in the previous study, there was a positive correlation between $\mathrm{IC}_{50}$ and the intensity of membrane HDM-2 expression in the AML stem cells. We did not observe this correlation in our present study. The $\mathrm{IC}_{50}$ for $\mathrm{U} 937$ cells was $4.7 \mu \mathrm{M}$ with cell surface HDM-2 fluorescence intensity of 22,000 while the $\mathrm{IC}_{50}$ for HL60 cells was $91.1 \mu \mathrm{M}$ with an intensity of 46,000 , two times the value for U937 cells. In other studies, we have also found that different isoforms of HDM-2 are expressed in the cell membranes of cancer cells (manuscriptforthcoming, Thadi et al. 2020), and possible differential expression of isoforms with varying affinities for PNC-27 may explain these differences between AML stem cells and more differentiated leukemia cell lines.

As found for solid tissue tumors and the AML cells in the previous study, we found that PNC-27 induces tumor cell necrosis in all three leukemia cell lines, and early or late 
stage apoptosis was not observed during PNC-27-induced cell killing. That PNC-27 induced pore formation in each of the three cell lines was indicated by the rapid release of $\mathrm{LDH}$, a cytosolic marker of necrosis, into the medium. Importantly, PNC-27-HDM-2 membrane interaction affects the half-life of this agent. Though the half-life of PNC-27 has been found in in vivo experiments to be in the order of $30 \mathrm{~min}$, but once bound to its membrane target HDM-2, its lifetime is substantially prolonged (10).

We also confirmed another feature of the tumoricidal effect of PNC-27 which is its independence of the status of p53 in cancer cells $(4,8)$. This gene has been shown to be mutated in more than $50 \%$ of human cancers, including leukemia (15). We found that PNC-27 kills a wide variety of solid tissue tumor cells in which p53 is homozygously deleted $(4,8)$. Two of our leukemia cell lines, U-937 that contains the DEL46A p53 null mutation (16) and HL-60 that has a biallelic deletion of p53 gene and is p53 null (17), were killed by PNC-27 which, as noted above, does not kill normal rat mononuclear cells at concentrations $(100 \mu \mathrm{M})$ above the $\mathrm{IC}_{50}$ values for PNC-27 on both cell lines. However, it likewise kills OCI-AML3, acute myelomonocytic leukemia cells with wildtype p53.

Acute myelogenous leukemia primarily affects a patient population of advanced age, with a median age at diagnosis of 68 years (18). Many patients have comorbidities and cannot tolerate cytotoxic chemotherapy. Recently several targeted, less toxic agents have become available to treat some patients with AML, including midastaurin and gilteritinib for FLT3+ patients, and ivosidenib and enasidenib for patients with IDH1 and IDH2 mutated leukemias. These treatments are limited, however, because only a minority of AML patients have these mutations, and many patients still relapse after treatment. Venetoclax, a BCL2 inhibitor that induces apoptosis, has a high level of activity, particularly when combined with a hypomethylating agent, but only about $60 \%$ of patients achieve a meaningful response and most ultimately relapse (19). Thus, there is significant need for additional, less toxic therapies in this patient population.

Our results indicate that $\mathrm{PNC}-27$ appears to be a promising agent with minimal toxicity. Clinical trials as a single agent or in combination with some of the other targeted medications should be pursued.

\section{Conflicts of Interest}

The Authors have no conflicts of interest related to this study.

\section{Authors' Contributions}

AT designed and conducted experiments, acquired and analyzed the data, wrote the article. LL, EG and MK conducted experiments, acquired, and analyzed the data. LS analyzed the data and reviewed the manuscript. BP conducted experiments. SS, MHB, KAW, MS and $\mathrm{PMC}$ reviewed the manuscript and provided administrative support. MRP and WBB designed experiments, analyzed the data, wrote the article and provided administrative support.

\section{Acknowledgements}

This work was performed in the Departments of Surgery and Pharmacology and Physiology at Drexel University College of Medicine. An unrestricted educational research grant was provided by Oncolyze Inc. Coauthor AA was awarded an American Medical Association student research grant for this research. Senior author WBB wishes to acknowledge and dedicate this research to our colleague at Drexel, Andres E. Castellanos, M.D., who passed away after a valiant struggle with leukemia.

\section{References}

1 Shaikh MF, Morano WF, Lee J, Gleeson E, Babcock BD, Michl J, Sarafraz-Yazdi E, Pincus MR and Bowne WB: Emerging role of mdm2 as target for anti-cancer therapy: A review. Ann Clin Lab Sci 46(6): 627-634, 2016. PMID: 27993876.

2 Wang H, Zhao D, Nguyen LX, Wu H, Li L, Dong D, Troadec E, Zhu Y, Hoang DH, Stein AS, Al Malki M, Aldoss I, Lin A, Ghoda LY, McDonald T, Pichiorri F, Carlesso N, Kuo YH, Zhang B, Jin J and Marcucci G: Targeting cell membrane HDM2: A novel therapeutic approach for acute myeloid leukemia. Leukemia 34(1): 75-86, 2020. PMID: 31337857. DOI: 10.1038/s41375-019-0522-9

3 Davitt K, Babcock BD, Fenelus M, Poon CK, Sarkar A, Trivigno V, Zolkind PA, Matthew SM, Grin'kina N, Orynbayeva Z, Shaikh MF, Adler V, Michl J, Sarafraz-Yazdi E, Pincus MR and Bowne WB: The anti-cancer peptide, PNC-27, induces tumor cell necrosis of a poorly differentiated non-solid tissue human leukemia cell line that depends on expression of hdm-2 in the plasma membrane of these cells. Ann Clin Lab Sci 44(3): 241248, 2014. PMID: 25117093.

4 Sarafraz-Yazdi E, Bowne WB, Adler V, Sookraj KA, Wu V, Shteyler V, Patel H, Oxbury W, Brandt-Rauf P, Zenilman ME, Michl J and Pincus MR: Anticancer peptide pnc-27 adopts an HDM-2-binding conformation and kills cancer cells by binding to hdm-2 in their membranes. Proc Natl Acad Sci USA 107(5): 19181923, 2010. PMID: 20080680. DOI: 10.1073/pnas.0909364107

5 Zhang J, Gu Y and Chen B: Mechanisms of drug resistance in acute myeloid leukemia. OncoTargets Ther 12: 1937-1945, 2019. PMID: 30881045. DOI: 10.2147/OTT.S191621

6 Alloush HM, Anderson E, Martin AD, Ruddock MW, Angell JE, Hill PJ, Mehta P, Smith MA, Smith JG and Salisbury VC: A bioluminescent microbial biosensor for in vitro pretreatment assessment of cytarabine efficacy in leukemia. Clin Chem 56(12): 1862-1870, 2010. PMID: 20921267. DOI: 10.1373/clinchem.2010.145581

7 Leung W, Hudson MM, Strickland DK, Phipps S, Srivastava DK, Ribeiro RC, Rubnitz JE, Sandlund JT, Kun LE, Bowman LC, Razzouk BI, Mathew P, Shearer P, Evans WE and Pui CH: Late effects of treatment in survivors of childhood acute myeloid leukemia. J Clin Oncol 18(18): 3273-3279, 2000. PMID: 10986060. DOI: $10.1200 /$ jco.2000.18.18.3273

8 Kanovsky M, Raffo A, Drew L, Rosal R, Do T, Friedman FK, Rubinstein P, Visser J, Robinson R, Brandt-Rauf PW, Michl J, Fine RL and Pincus MR: Peptides from the amino terminal 
mdm-2-binding domain of $\mathrm{p} 53$, designed from conformational analysis, are selectively cytotoxic to transformed cells. Proc Natl Acad Sci USA 98(22): 12438-12443, 2001. PMID: 11606716. DOI: $10.1073 /$ pnas. 211280698

9 Rosal R, Pincus MR, Brandt-Rauf PW, Fine RL, Michl J and Wang $\mathrm{H}$ : Nmr solution structure of a peptide from the $\mathrm{mdm}-2$ binding domain of the p53 protein that is selectively cytotoxic to cancer cells. Biochemistry 43(7): 1854-1861, 2004. PMID: 14967026. DOI: $10.1021 / \mathrm{bi035718g}$

10 Sookraj KA, Bowne WB, Adler V, Sarafraz-Yazdi E, Michl J and Pincus MR: The anti-cancer peptide, pnc-27, induces tumor cell lysis as the intact peptide. Cancer Chemother Pharmacol 66(2): 325-331, 2010. PMID: 20182728. DOI: 10.1007/s00280-009$1166-7$

11 Do TN, Rosal RV, Drew L, Raffo AJ, Michl J, Pincus MR, Friedman FK, Petrylak DP, Cassai N, Szmulewicz J, Sidhu G, Fine RL and Brandt-Rauf PW: Preferential induction of necrosis in human breast cancer cells by a p53 peptide derived from the mdm2 binding site. Oncogene 22(10): 1431-1444, 2003. PMID: 12629507. DOI: 10.1038/sj.onc. 1206258

12 Jensen HA, Yourish HB, Bunaciu RP, Varner JD and Yen A: Induced myelomonocytic differentiation in leukemia cells is accompanied by noncanonical transcription factor expression. FEBS Open Bio 5: 789-800, 2015. PMID: 26566473. DOI: 10.1016/j.fob.2015.09.008

13 Hollink IHIM, Feng Q, Danen-van Oorschot AA, ArentsenPeters STCJM, Verboon LJ, Zhang P, de Haas V, Reinhardt D, Creutzig U, Trka J, Pieters R, van den Heuvel-Eibrink MM, Wang $J$ and Zwaan CM: Low frequency of dnmt3a mutations in pediatric aml, and the identification of the oci-aml3 cell line as an in vitro model. Leukemia 26(2): 371-373, 2012. PMID: 21836609. DOI: 10.1038/leu.2011.210
14 Allende-Vega N and Villalba M: Metabolic stress controls mutant p53 r248q stability in acute myeloid leukemia cells. Sci Rep 9(1): 5637-5637, 2019. PMID: 30948782. DOI: 10.1038/s41598-019-42220-y

15 Sigal A and Rotter V: Oncogenic mutations of the p53 tumor suppressor: The demons of the guardian of the genome. Cancer Res 60(24): 6788-6793, 2000. PMID: 11156366.

16 Shah A and Seedhouse C: Frequency of tp53 mutations and its impact on drug sensitivity in acute myeloid leukemia? Indian J Clin Biochem 27(2): 121-126, 2012. PMID: 23543587. DOI: 10.1007/s12291-012-0203-1

17 Leroy B, Girard L, Hollestelle A, Minna JD, Gazdar AF and Soussi T: Analysis of tp53 mutation status in human cancer cell lines: A reassessment. Hum Mutat 35(6): 756-765, 2014. PMID: 24700732. DOI: $10.1002 /$ humu. 22556

18 American Cancer Society 2020: Key statistics for acute myeloid leukemia (AML). Available at: https://www.cancer.org/cancer/ acute-myeloid-leukemia/about/key-statistics.html [Last accessed May 28, 2020]

19 DiNardo CD, Pratz K, Pullarkat V, Jonas BA, Arellano M, Becker PS, Frankfurt O, Konopleva M, Wei AH, Kantarjian HM, Xu T, Hong WJ, Chyla B, Potluri J, Pollyea DA and Letai A: Venetoclax combined with decitabine or azacitidine in treatmentnaive, elderly patients with acute myeloid leukemia. Blood 133(1): 7-17, 2019. PMID: 30361262. DOI: 10.1182/blood2018-08-868752 